\title{
Ochratoxine A et néphropathie humaine en Tunisie : dix ans d'étude
}

\author{
Ochratoxin A and human nephropathy in \\ Tunisia : a ten year survey
}

\author{
Wafa HASSEN $^{(1)}$, Salwa ABID-ESSEFI ${ }^{(1)}$, Abdellatif ACHOUR ${ }^{(1,2)}$, \\ Khira MAAROUFI ${ }^{(3)}$, Edmond CREPPY(4), Hassen BACHA*(1)
}

(1) Laboratoire de Recherche sur les Substances Biologiquement Compatibles (LRSBC), Faculté de Médecine Dentaire, Rue Avicenne - 5019 MONASTIR - TUNISIE

(2) Service de Néphrologie, CHU et Faculté de Médecine, Rue Avicenne - 5019 MONASTIR - TUNISIE

(3) Laboratoire de Biochimie, Faculté de Pharmacie, Rue Avicenne - 5019 MONASTIR - TUNISIE

(4) Laboratoire de Toxicologie et d'Hygiène Appliquée, UFR des Sciences Pharmaceutiques, Université

Bordeaux II, 146, Rue Léo Saignat - $\$ 3076$ BORDEAUX Cedex - FRANCE

* Auteur à qui adresser la correspondance : Professeur Hassen BACHA, Directeur du Laboratoire de Recherche sur les Substances Biologiquement Compatibles (LRSBC), Faculté de Médecine Dentaire, Rue Avicenne - 5019 MONASTIR - TUNISIE

Tél : +21698296780 - Fax : +21673461150 - E-mail : hassen.bacha@fmdm.rnu.tn

\section{$R E ́ S U M E$}

L'ochratoxine A (OTA) est une mycotoxine connue essentiellement pour ses effets néphrotoxiques. Des études réalisées dans les régions balkaniques ont fortement soupçonné l'OTA d'être le principal agent causal de la Néphropathie Endémique des Balkans (NEB). Cependant, malgré les nombreuses investigations réalisées, l'implication de l'OTA dans cette néphropathie humaine est encore sujette à controverse. En Tunisie, une néphropathie interstitielle chronique (NIC) à étiologie indéterminée, similaire à la NEB, où l'OTA semble également être impliquée, a été bien caractérisée. Dans ce travail, nous nous proposons d'apporter des preuves supplémentaires impliquant davantage l'OTA dans cette néphropathie. Nous présentons le bilan d'une étude rétrospective de dix ans (1991-2001) engageant 954 néphropathes et 205 individus sains. Dans ce bilan, nous comparons les contaminations sériques en OTA dans trois groupes: un groupe d'individus sains ne présentant aucune pathologie (205 sujets),

\section{SUMMARY}

Ochratoxin A (OTA) is a mycotoxin essentially known for its nephrotoxic effects. Studies performed in the Balkans pointed out OTA as the main causal agent of Balkan Endemic Nephropathy (BEN). However, despite the considerable amount of data regarding this disease, it is still controversial whether OTA plays a causative or only a subordinate role in the induction of this human nephropathy.

In Tunisia, a chronic interstitial nephropathy (CIN) of unknown cause, perfectly similar to BEN, was well characterised and wherein OTA seems to be also implicated. In this study, we tried to consolidate the suspected link between blood OTA contamination and Tunisian Chronic Interstitial Nephropathy (CIN) of unknown aetiology. Indeed, we give hereby the assessment of a 10-years retrospective study (1991-2001) including 954 nephropathy patients and 205 healthy subjects. In this survey, we have compared frequency of positive samples, the means and the range of blood 
un groupe de néphropathes présentant une NIC inexpliquée (383 sujets) et un groupe de néphropathes à étiologie connue (571 sujets). La détection et le dosage de l'OTA sont réalisés après extraction à partir des échantillons sériques suivie d'une analyse par chromatographie liquide haute performance (HPLC) avec détection de fluorescence. Nous montrons que l'incidence la plus importante de la contamination sérique en OTA est trouvée dans le groupe présentant une NIC inexpliquée. En effet, dans ce groupe, le pourcentage d'individus OTA-positifs est de $97 \%$ et il n'est que de $73 \%$ et de $86 \%$ respectivement chez les individus sains et chez ceux présentant d'autres néphropathies à étiologie connue. Les concentrations moyennes sont également plus élevées dans le groupe de NIC d'étiologie inconnue (50.77 \pm 4.75 $\mathrm{ng} / \mathrm{ml})$ que dans les autres groupes de contrôle $(2.35 \pm 0.90$ $n g / m l)$ ou de néphropathes à étiologies connues (9.96 \pm 3 $\mathrm{ng} / \mathrm{ml}$ ). Notre étude renforce davantage le rôle de l'OTA dans cette néphropathie et la désigne comme agent causal très probable.

\section{MOTS-CLÉS}

Ochratoxine A, Sang, HPLC, Néphropathie Humaine, Tunisie.

\section{Introduction}

L'ochratoxine A (OTA) (Figure 1) est une mycotoxine produite par des champignons des genres Aspergillus et Penicillium $(1,2)$. Elle est transmise à l'homme par l'intermédiaire de la chaîne alimentaire habituellement contaminée par cette toxine. $(3,4,5)$.

L'OTA a de multiples effets toxiques. En effet, plusieurs études ont montré qu'elle est tératogène (6), immunosuppressive (7) ; elle inhibe la synthèse protéique (8) et altère plusieurs paramètres biochimiques (9). Par ailleurs, elle présente des effets génotoxiques et mutagènes sur des cellules en culture $(10,11)$.

Parmi ces toxicités, la néphrotoxicité apparaît la plus importante $(12,13)$. Des études ont montré que l'OTA induit des adénomes rénaux chez la souris et chez le rat $(14,15)$; de plus, l'OTA apparaît comme la cause principale de la néphrotoxicité porcine spontanée et de néphropathie chez les volailles $(16,17)$.

La néphropathie endémique des Balkans (NEB) est une néphropathie chronique tubulointerstitielle décrite pour la première fois en 1957. Malgré les nombreuses investigations réalisées, son étiologie reste un problème majeur non résolu (18). Plusieurs agents étiologiques ont été proposés : le plomb (19), le cadmium (20), l'acide aristolochique (21), les hydrocarbures aromatiques polycycliques (22), la carence en sélénium (23) etc..., mais aucun n'a été définitivement retenu. Cependant, un certains nombre d'arguments sont en faveur de l'implication de l'OTA dans la genèse de la NEB. Ces arguments sont les suivants :
OTA contamination in three groups : a group of healthy subjects with no pathology (205 subjects), a group presenting CIN of unknown cause (381 subjects) and a group with other chronic kidney disease of known cause (571 subjects). OTA detection and assay were performed after serum samples extraction followed by high performance liquid chromatography (HPLC) analysis with fluorescence detection. Our results show that the highest incidence was found in CIN of unknown cause group. Indeed, in this group, the percentage of OTA-positive samples is up to $97 \%$ whereas it is only $73 \%$ and $86 \%$ respectively in control and in other kidney disease groups. The mean concentrations are also highest in CIN of unknown cause group $(50.77 \pm 4.75 \mathrm{ng} / \mathrm{ml})$ as opposed to controls $(2.35 \pm 0.90 \mathrm{ng} / \mathrm{ml})$ and other chronic kidney diseases group $(9.96 \pm 3 \mathrm{ng} / \mathrm{ml})$.

Our study emphasizes further the implication of OTA in this particular human nephropathy and underlines the probable causative role of OTA in the onset of this disease.

\section{KEY-WORDS}

Ochratoxin A, Blood, HPLC, Human Nephropathy, Tunisia.

- La NEB présente des ressemblances frappantes sur les plans biologique, histologique et radiologique avec la néphropathie porcine induite naturellement par l'OTA par l'intermédiaire de l'alimentation (24).

- Une néphropathie interstitielle chronique identique en tout point à la NEB est induite sur plusieurs modèles animaux intoxiqués expérimentalement par la toxine (rat, souris, volailles, chien, ... etc.) $(12,25)$.

- L'association de la NEB à l'apparition des tumeurs du tractus urinaires est également trouvée dans les cas de néphropathie expérimentalement induite par l'OTA $(26,27)$.

- Des adduits qui sont des complexes covalents de la toxine et des bases de l'ADN ont été détectés chez des patients présentant la NEB. Ces adduits sont identiques à ceux révélés chez les animaux ayant développé expérimentalement une néphropathie induite par l'OTA (28, 29).

- Dans les régions endémiques (Bulgarie, exYougoslavie, Roumanie,...), des niveaux de contamination très élevés en OTA ont été détectés dans les céréales, dans les tissus d'animaux ainsi que dans le sang humain (26).

Pour toutes ces raisons l'OTA a été proposée comme agent étiologique causal très probable de la NEB (18, 27).

Les données accumulées jusqu'à maintenant indiquent que l'origine de la NEB serait environnementale et appuient l'implication de cette mycotoxine dans la genèse de cette néphropathie humaine $(30,31)$.

Cependant, aucune évidence ne montre le lien direct 


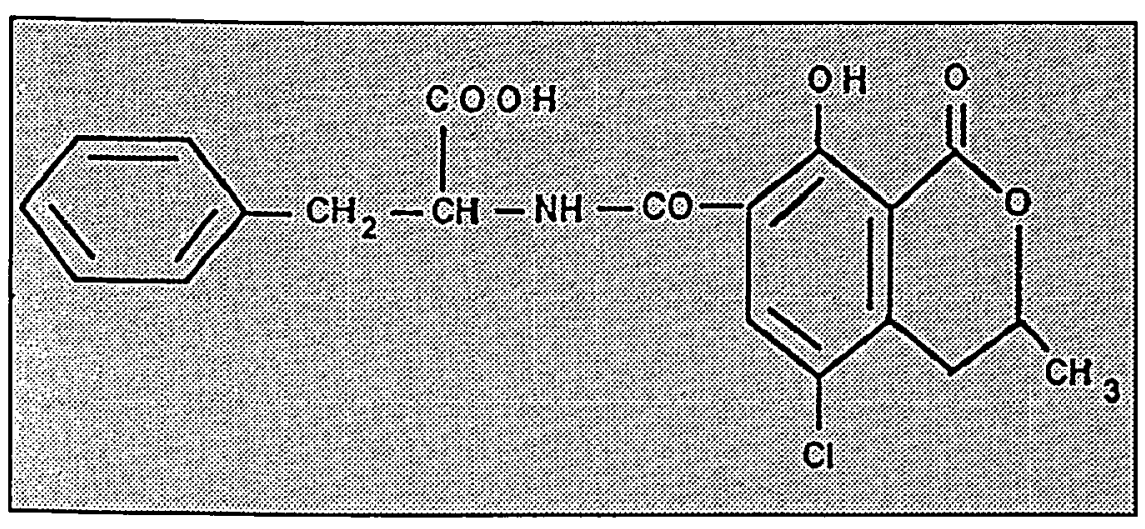

Figure 1 : Structure Chimique de l'Ochratoxine A.

entre l'OTA et cette néphropathie. En effet, ce type de néphropathie est caractérisé par son évolution lente. Il s'écoule un temps long entre la période de la contamination et le moment d'apparition de la maladie. De plus, les niveaux de contamination sérique élevés chez les insuffisants rénaux peuvent s'expliquer par l'accumulation de la toxine et non par son implication dans la survenue de la maladie. Par ailleurs, la relation causeeffet n'est pas évidente dans la mesure où dans des populations saines, des personnes peuvent présenter des contaminations par la toxine - qui restent tout de même bas - sans pour autant développer la néphropathie.

Des travaux récents montrent que d'autres populations dans d'autres régions du monde sont aussi exposées à l'OTA (32). La Tunisie est également concernée. Les premières investigations ont montré la présence de l'OTA dans une large variété d'aliments fréquemment consommés (4). Une néphropathie interstitielle chronique (NIC) à étiologie indéterminée, similaire à la NEB, a été bien caractérisée $(33,34)$. Par ailleurs, chez les populations néphropathes, l'OTA a été détectée à des niveaux importants dans le sang (35). De plus, l'origine de la contamination semble exclusivement alimentaire (36). Compte tenu de la similitude de la NEB et de la NIC, trouvée en Tunisie, notre intérêt s'est focalisé sur la responsabilité effective de l'OTA dans la genèse de ces deux néphropathies qui peuvent finalement n'en faire qu'une. Ainsi, une étude épidémiologique sur dix années (1991-2001) a été réalisée, où l'OTA a été systématiquement recherchée et dosée dans un grand nombre d'échantillons sériques d'individus sains et de néphropathes présentant une NIC à étiologie indéterminée et d'individus présentant d'autres néphropathies à étiologie connue.

Dans cet article, nous rapportons le bilan de cette étude rétrospective et nous proposons une corrélation nette entre les niveaux de contamination sérique en OTA et la NIC à étiologie indéterminée. Nos résultats appuient fortement l'implication de l'OTA dans la genèse de cette néphropathie et confortent les travaux désignant l'OTA comme agent causal de cette néphropathie humaine.

\section{Matériel et méthodes}

\section{Matériel :}

\section{Produits chimiques}

- L'ochratoxine A standard provient de Sigma Chemicals (St Louis, Mo, USA), elle est dissoute dans du méthanol. La concentration de l'OTA dans ce solvant est déterminée spectrophotométriquement $(\lambda$ d'excitation $=330 \mathrm{~nm}$, coefficient d'extinction molaire de l'OTA dans le méthanol $\varepsilon=5500$ ).

- Colonne HPLC (ODS II, $\mathrm{C}_{18}$, porosité $10 \mu \mathrm{m}, 250 \times 8$ $\mathrm{mm})$ et cartouches Sep-pak $\mathrm{C}_{18}(20 \times 10 \mathrm{~mm})$, (Superlco, Sigma-Aldrich Chimie, France).

- Les autres produits et solvants proviennent de Merck, de Prolabo et de Sigma-Aldrich, (France) et sont d'un haut degré de pureté.

\section{Matériel Biologique}

Les néphropathes ont été recrutés dans les différents centres hospitaliers des chefs-lieux des principales régions de Tunisie : la région du nord-ouest (Jendouba), la région $\mathrm{d}$ nord-est (Tunis), la région du centre (Monastir), la région du sud-ouest (Gafsa) et la région du sud-est (Sfax). Les témoins sont des donneurs volontaires ne présentant aucune pathologie, ils sont recrutés dans les mêmes régions. Il s'agit dans tous les cas de sujets âgés de 40 à 80 ans avec une parité homme-femme pour chaque groupe considéré. Leurs habitudes alimentaires sont proches. Les aliments de base sont ceux de la chaîne alimentaire tunisienne classique (céréales, semoules, couscous, pâtes alimentaires, fruits et légumes secs, ...etc.) avec cependant certaines particularités régionales de préparation et de conservation. Un des critères d'inclusion dans la sélection des sujets néphropathes est la créatininémie qui doit être entre 200 et $600 \mu \mathrm{M}$.

\section{Méthodes :}

\section{Choix des patients}

Les sujets choisis sont répartis en 3 groupes principaux : - un groupe de néphropathes chroniques (571 sujets) présentant diverses néphropathies : néphropathie glomérulaire chronique (NGC) ; néphropathie vasculaire chronique (NVC) ; néphropathie interstitielle chronique (NIC) ou autres néphropathies. Pour tous ces cas, les étiologies sont connues.

- un groupe de néphropathes interstitielle chronique (NIC) à étiologie indéterminée (383 individus), sélectionnés selon des critères d'inclusion (âge, créatininémie, examen biologique et radiologique) et des critères d'exclusion (néphropathies non interstitielles, néphropathies diabétiques et néphropathies interstitielles d'étiologie connue). 
- un groupe de contrôle (205 individus) ne présentant aucune pathologie.

Pour chaque personne recrutée, patients ou témoins, un rapport médical a été élaboré, comportant les indications suivantes :

- l'âge, le sexe, l'origine géographique, la profession

- les habitudes alimentaires, la nature et l'état des aliments fréquemment consommés

- les prises éventuelles de médicaments

- les antécédents pathologiques personnels et familiaux.

\section{Prélèvements}

Pour tous les individus, nous avons prélevé des échantillons de sang $(7 \mathrm{ml})$ pour le dosage de l'OTA. Des échantillons d'urine $(5 \mathrm{ml})$ ont été également prélevé et ont servi à l'évaluation biologique de la fonction rénale.

\section{Extraction et dosage de l'OTA}

L'extraction de l'OTA à partir des échantillons sériques a été réalisée selon Bauer et Gareis (37) et Bacha et ses collaborateurs (5). Les échantillons de sang recueillis sur des tubes secs sont centrifugés à $2000 \mathrm{~g}$ pendant 15 min. à $4^{\circ} \mathrm{C}$. Les sérums obtenus $(3 \mathrm{ml})$ sont ensuite acidifiés avec l'acide acétique $1 \mathrm{M}$ jusqu'à atteindre le $\mathrm{pH}$ 4,5 , ils sont nettoyés sur des cartouches Sep-Pack, waters $\mathrm{C} 18$. Ce nettoyage se déroule par lavage à l'acide acétique $2 \%(\mathrm{v} / \mathrm{v})(7,5 \mathrm{ml})$, puis élution de l'OTA avec un mélange méthanol, $\mathrm{HCl} 1 \%(\mathrm{v} / \mathrm{v})(10 \mathrm{ml})$. Les éluats sont ensuite purifiés par HPLC en phase inverse. L'élution de l'OTA de la colonne HPLC se fait par le mélange méthanol : acétonitrile : acétate de sodium 5mM : acide acétique (300:300:400:14, v/v/v/v, $\mathrm{pH}=7,2)$ à un débit de $1 \mathrm{ml} / \mathrm{min}$ pendant $15 \mathrm{~min}$. La détection se fait par fluorescence, longueur d'onde d'excitation $=340 \mathrm{~nm}$ et longueur d'onde émission = $465 \mathrm{~nm}$. Le pic correspondant à l'OTA apparaît à un temps de rétention de 6,3 min (Figure 2). La quantification est réalisée par comparaison à des standards d'OTA de concentrations connues injectés dans les mêmes conditions en utilisant un logiciel d'acquisition et de traitement des chromatogrammes (Normasoft, ICS France) qui intègre les surfaces sous les pics. Le rendement de l'extraction de l'OTA par cette méthode est d'environ $85 \%$. La détection est linéaire avec un coefficient de corrélation $\mathrm{R}=0,99967$. La limite de détection est de $0,1 \mathrm{ng} / \mathrm{ml}$.

Dans un souci de reproductibilité, chaque échantillon est chromatographié à deux reprise. Pour s'assurer qu'il s'agit bien de l'OTÁ, nous procédons à sa confirmation soit par dérivation soit par hydrolyse acide suivie d'une détection des deux composants de l'OTA, l'ochratoxine $\alpha$, OT $\alpha$ et la phénylalanine (36).

\section{Investigations cliniques}

Le but essentiel des investigations cliniques est la détermination du type anatomo-clinique de la néphropathie, cette détermination est basée d'une part sur les antécédents pathologiques et d'autre part sur un ensemble de signes :

- signes cliniques : hypertension artérielle, œdème, hématurie, troubles urinaires, lombalgies, qui constituent les critères d'exclusion.

- signes radiologiques incluant l'échographie rénale et l'artériographie.

- signes biologiques qui permettent de distinguer avec certitude les différents types de néphropathie (sédiments urinaires, protéinurie, ionogramme urinaire, électrophorèse des protéines).

- occasionnellement des investigations anatomo-pathologiques ont été réalisées mais de manière non systématique, dans le but de confirmer le diagnostic.

Le diagnostic de la NIC à étiologie indéterminée est un diagnostic réalisé par élimination après avoir écarté toute cause de néphropathie interstitielle chronique.

\section{Analyses statistiques}

Les niveaux de contamination par l 'OTA obtenus après dosage dans les trois groupes sont comparés entre eux et comparés à celui du groupe témoin en utilisant un test statistique non paramétrique (Wilcoxon Rank-Sum Test) (38). Les résultats sont exprimés par la moyenne plus ou moins la déviation standard.

\section{Résultats}

Le tableau I donne les pourcentages d'individus présentant l'OTA dans le sang (OTA-positifs), les concentrations moyennes d'OTA ainsi que les valeurs limites dans les trois groupes étudiés. Nous avons trouvé que $73 \%$ de la population générale saine étudiée présente une contamination sérique en OTA avec une concentration moyenne de $2,35 \pm 0,90 \mathrm{ng} / \mathrm{ml}$ au seuil de détection de $0,1 \mathrm{ng} / \mathrm{ml}$. Concernant les sujets présentant une NIC inexpliquée, le pourcentage d'individus OTApositifs est de l'ordre de $97 \%$ et la concentration moyenne est de 50,77 $\pm 4,75 \mathrm{ng} / \mathrm{ml}$. Pour les individus présentant des néphropathies chroniques (glomérulaire, vasculaire ou interstitielle) d'étiologie connue, $86 \%$ d'entre eux sont OTA-positifs avec une concentration moyenne de $9,96 \pm 3 \mathrm{ng} / \mathrm{ml}$. La comparaison statistique entre ces deux groupes montre que la différence est significative à $\mathrm{p}<0,001$. Les valeurs limites sont corrélées à l'incidence et à la concentration moyenne, en effet, elles sont aussi plus élevés chez les individus présentant une NIC inexpliquée avec une valeur maximale de $140,5 \mathrm{ng} / \mathrm{ml}$. 


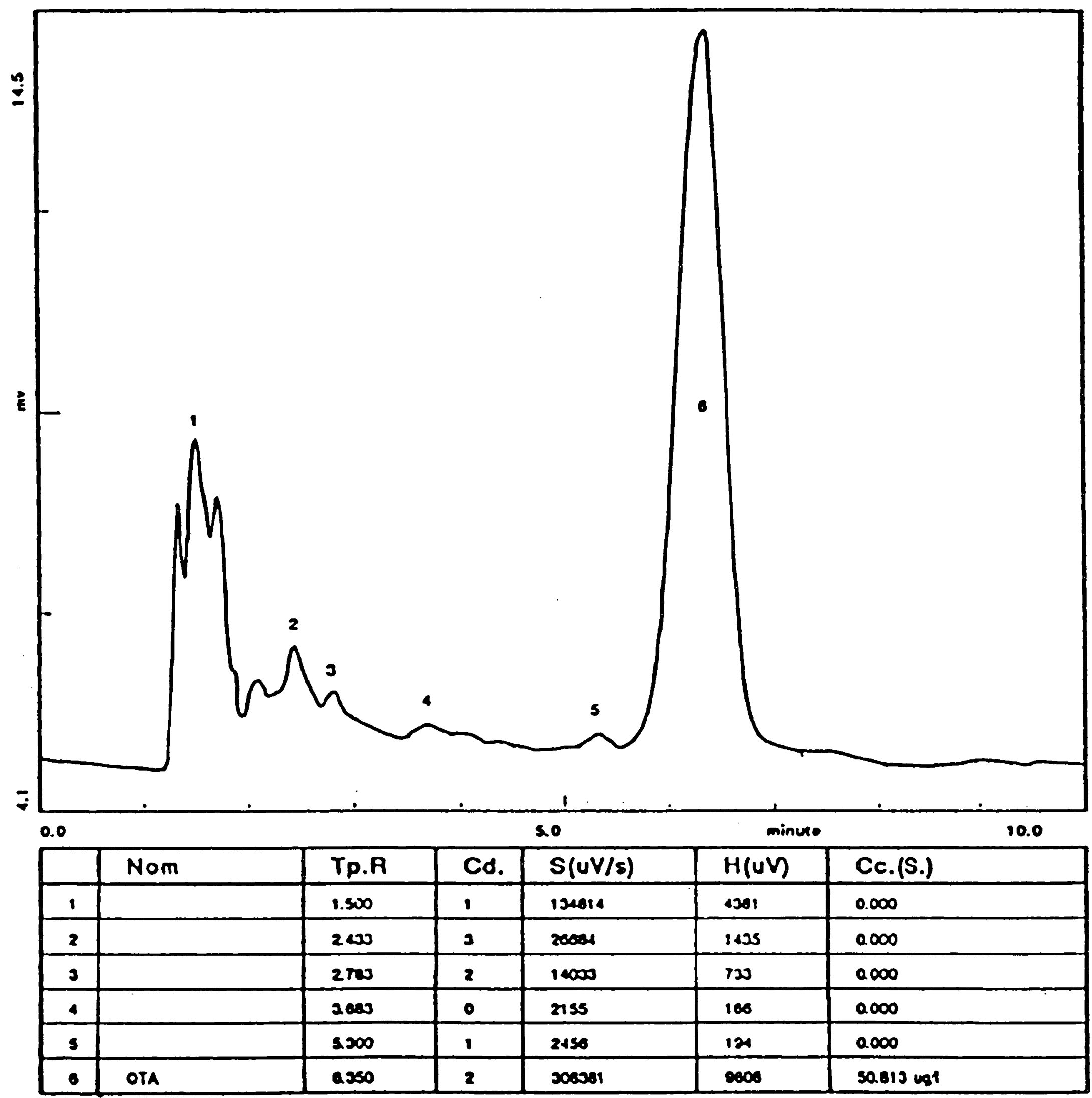

Figure 2 : Chromatogramme d'un extrait sérique d'un patient présentant la NIC d'étiologie indéterminée.

Tableau I : Pourcentage d'échantillons de sang (OTA-positifs), valeurs limites et concentrations moyennes d'OTA dans le sang chez une population générale témoin, chez une population présentant une néphropathie interstitielle chronique (NIC) inexpliquée et chez une population présentant des néphropathies chroniques diverses d'étiologie connue.

\begin{tabular}{|c|c|c|c|}
\hline & & \multicolumn{2}{|c|}{ Néphropathes } \\
\cline { 2 - 4 } & $\begin{array}{c}\text { Individus } \\
\text { Sains }\end{array}$ & $\begin{array}{c}\text { NIC } \\
\text { inexpliquée }\end{array}$ & $\begin{array}{c}\text { Autres } \\
\text { néphropathies }\end{array}$ \\
\hline Nombre d'individus & 205 & 383 & 571 \\
\hline Incidence (\%) & 73 & 97 & 86 \\
\hline Moyenne \pm D.S. (ng/ml) & $2,35 \pm 0,90$ & $50,77 \pm 4,75$ a & $9,96 \pm 3$ \\
\hline Valeurs Limites (ng/ml) & $0-7,5$ & $1,74-140,5$ & $0-29$ \\
\hline
\end{tabular}

a : significativement différent du groupe d'individus présentant diverses néphropathies d'étiologie connue à $\mathrm{p}<0,001$.
Ainsi, on peut dire que les concentrations d'OTA les plus élevées sont trouvées chez les individus présentant une NIC inexpliquée. Chez l'autre groupe de néphropathes, bien que l'OTA soit présente, sa concentration reste relativement faible et non significativement différente de celle du groupe témoin.

\section{Discussion}

Les mycotoxines et les pathologies induites représentent de sérieux problèmes économiques et sanitaires dans plusieurs pays du monde (39). Nos travaux antérieurs ont montré la présence de mycotoxines diverses 
en Tunisie, y compris l'ochratoxine A. Cette présence serait liée aux caractéristiques climatiques et géographiques de la Tunisie ainsi qu'aux conditions socioéconomiques de sa population (40).

L'inventaire et l'analyse des aliments courants de la chaîne alimentaire tunisienne (céréales et dérivés, semoules, couscous, pâtes alimentaires, aliments composés à base de céréales, viandes et poissons séchés, ...etc.) a montré que 70 à $90 \%$ des aliments fréquemment consommés sont contaminés par diverses mycotoxines, souvent à des concentrations très élevées (4). Cela a pour conséquence une transmission de certaines de ces mycotoxines à l'homme. Une corrélation entre la présence de ces mycotoxines dans les aliments et des pathologies spécifiques a été mise en évidence (41).

L'ochratoxine A est omniprésente en Tunisie. Sa prévalence paraît plus élevée qu'en Europe, en effet 62 à $82 \%$ de la population générale présentent l'OTA dans le sang à un seuil de détection de $0,1 \mathrm{ng} / \mathrm{ml}(40,41)$.

Une ressemblance entre la néphropathie interstitielle chronique (NIC) à étiologie indéterminée caractérisée en Tunisie et la néphropathie endémique des Balkans (NEB) a été relevée. En effet, l'analogie en tout point entre ces deux types de néphropathies est remarquable. Pour la NEB, l'agent causal très fortement désigné est l'ochratoxine A (42-45). Compte tenu de la forte prévalence de l'OTA dans la chaîne alimentaire tunisienne, nous avons pensé que cette NIC à étiologie indéterminée serait, à l'instar de la NEB, induite par l'OTA. Ainsi, dans le but de dégager une corrélation entre les taux sériques d'OTA et la NIC à étiologie indéterminée et d'appuyer davantage l'implication de l'OTA dans ce type de néphropathie humaine, nous avons évalué pendant une dizaine d'années, la contamination sérique en OTA chez des individus sains, chez des individus à NIC inexpliquée et chez des individus présentant d'autres types de néphropathies chroniques d'étiologie connues. Nous avons pu montrer que les niveaux de contamination en OTA sont significativement $(\mathrm{p}<0,001)$ plus élevés chez les néphropathes à NIC inexpliquée que chez les autres types de néphropathies à étiologie connue où les niveaux de contaminations sont comparables à ceux trouvés chez la population générale.

Il en ressort de ce résultat que l'association révélée entre les niveaux de contamination sérique en OTA et la survenue de la NIC à étiologie indéterminée n'est pas liée au hasard puisque cette association est vérifiée sur une longue période (10 ans) et a porté sur un grand nombre d'échantillons (954 néphropathes). Il s'agit d'un échantillonnage représentatif, en effet, le rapport du Ministère de la Santé de Tunisie indique un nombre global de néphropathes de 9570 (46) ; notre étude a donc porté sur environ $10 \%$ de néphropathes.
Par ailleurs, nous sommes en mesure d'écarter l'hypothèse selon laquelle les niveaux sériques d'OTA élevés trouvés chez les néphropathes seraient la conséquence de l'insuffisance rénale et non pas la cause de sa survenue. En effet, si cela était le cas, nous aurions trouvé les mêmes niveaux de contamination sériques indifféremment chez tous les insuffisants rénaux étudiés alors que ces niveaux sont clairement et significativement différents entre le groupe NIC à étiologie indéterminée et le groupe présentant d'autres néphropathies d'étiologie connue.

Nos résultats confortent davantage le rôle de l'OTA dans la genèse de cette néphropathie humaine puisque la toxine est trouvée associée à la néphropathie dont l'étiologie est indéterminée ; elle pourrait être, de ce fait, désignée comme agent étiologique causal. Si l'OTA était trouvée associée à une néphropathie d'étiologie connue, elle serait alors considérée comme un facteur aggravant uniquement.

Compte tenu de l'ensemble des données accumulées incluant nos présents arguments et même en l'absence encore de preuves directes, nous pouvons appuyer fortement l'implication de cette mycotoxine dans la survenue de la néphropathie interstitielle chronique d'étiologie indéterminée.

Il est important de noter que les niveaux de contamination trouvés chez la population à NIC inexpliquée sont plus importants que ceux trouvés chez la population endémique balkanique comme le montre une étude similaire réalisée sur 6910 individus pendant dix ans dont 4343 individus originaires de Kaniza, région hyperendémique des Balkans où les valeurs limites de contamination sont de 2 à $50 \mathrm{ng} / \mathrm{ml}$ alors qu'en Tunisie ces valeurs vont de 1,74 à $140,5 \mathrm{ng} / \mathrm{ml}$ (47).

Concernant la population générale tunisienne saine, les niveaux de contamination sont plus importants que ceux trouvés dans des régions non endémiques comme par exemple en Italie, l'OTA est présente avec une moyenne de $0.56 \mathrm{ng} / \mathrm{ml}$ et des valeurs limites allant de 0,12 à $2,84 \mathrm{ng} / \mathrm{ml}$ (48). La Turquie, autre région non endémique, des taux de contamination de $0,4 \mathrm{ng} / \mathrm{ml}$ et des valeurs limites allant de 0,19 à $1,43 \mathrm{ng} / \mathrm{ml}$ (49). De même, les individus sains de la région balkanique mais non endémique présentent des taux de contamination beaucoup moins élevés que ceux trouvés chez les individus sains tunisiens (tableau II). Cette situation est sans doute liée aux valeurs de « daily intake » ou quantité journalière disponible en OTA qui sont très élevées en Tunisie, elles se situent entre 4,7 et $130 \mathrm{ng} / \mathrm{kg}$ corporel / jour (5) alors que les valeurs tolérées et recommandées par le EC's Scientific Commitee for Food (SCF) ne sont que de $5 \mathrm{ng} / \mathrm{kg}$ corporel/ jour (50). Ainsi, la disponibilité de l'OTA dans la chaîne alimentaire en Tunisie pourrait-être de 25 fois supérieure aux normes. 
Tableau II : Comparaison des valeurs limites et des concentrations moyennes d'OTA chez des individus sains, en Tunisie, aux Balkans (dans une zone non endémique), en Italie et en Turquie (pays non endémique).

\begin{tabular}{|l|c|c|c|c|}
\hline & Tunisie & Balkans & Italie & Turquie \\
\hline Nombre d'individus sains & 205 & 2566 & 138 & 97 \\
\hline Moyenne \pm D.S. $(\mathrm{ng} / \mathrm{ml})$ & $2,35 \pm 0,90$ & $2,125 \pm 3,64$ & 0,56 & $0,4 \pm 0,28$ \\
\hline Valeurs Limites $(\mathrm{ng} / \mathrm{ml})$ & $0-7,5$ & $0-10$ & $0,12-2,48$ & $0,19-1,43$ \\
\hline
\end{tabular}

\section{Conclusion}

Ainsi, en Tunisie, l'OTA a été trouvée, à des taux plus élevés et de manière significative $(p<0,001)$, dans un groupe représentatif développant une néphropathie interstitielle chronique de cause inconnue que dans d'autres groupes de contrôle ou de néphropathies d'étiologie connue. Cela fait de l'OTA un agent étiologique très probable pour ce type de néphropathie inexpliquée.

Par ailleurs, la situation en Tunisie est similaire à celle des régions balkaniques, elle présente même des niveaux de contamination plus élevés. Ces données désignent la Tunisie comme un second foyer de néphropathie endémique.
Malgré l'absence d'évidences impliquant d'une manière directe l'OTA dans ce type de néphropathie, les données obtenues jusqu'à maintenant, aussi bien dans les régions balkaniques qu'en Tunisie désignent l'OTA comme agent causal très probable.

En l'absence de preuves directes impliquant l'OTA dans cette néphropathie et comme aucune évidence ne va à l'encontre de cette hypothèse, l'OTA reste l'agent étiologique le plus probable. Ainsi, une attention particulière doit être accordée à l'exposition humaine à cette mycotoxine. Des prospections continues de la présence de cette mycotoxine dans la chaîne alimentaire doivent être régulièrement réalisées.

\section{Remerciements :}

Ce travail a été soutenu par le Ministère Tunisien de la Recherche Scientifique et de la Technologie (Laboratoire de Recherche sur les Substances Biologiquement Compatibles, LRSBC) et par le Ministère Français des Affaires Etrangères et la Direction Générale de la Recherche Scientifique DGRST (Action intégrée de Coopération Interuniversitaire Franco-Tunisienne, CMCU 01 F0904).

\section{Références}

1. Van der Merwe K.J., Steyn P.S., Fourie L., Scott D.B., Theron, J.J. Ochratoxin A, a toxic metabolite produced by Aspergillus ochraceus Wilh. Nature. $1965 ; 205$ : 1112-1113.

2. Steyn P.S. Ochratoxins and related dihydroisocoumarins. In: Betina, V., ed. Mycotoxins-Production, Isolation, Separation and Purification. Elsevier, Amesterdam, 1984 ; 183-216.

3. Krogh P. Ochratoxins in food. In : Krogh, P., ed. Mycotoxins in Food. Harcourt Brace Jovanovich, London, 1987 ; 97-121.

4. Bacha H., Hadidane R., Regnault C., Ellouz F. Creppy E.E. and Dirheimer G. Monitoring and Identification of Fungal Toxins in Food Products, Animals Feed and Cereals in Tunisia. Journal of Stored Product Research. $1988 ; 4: 199-206$.

5. Bacha H., Maaroufi K., Achour A., Hammami M., Ellouz F. and Creppy E.E. Ochratoxines et Ochratoxicoses Humaines en Tunisie. In: Creppy, E.E., Castegnaro, M., Dirheimer, G. (Eds.), Human Ochratoxicosis and its Pathologies, vol. 231. Colloque INSERM/John Libbey Eurotext, 1993 ; 111-121.

6. Mayura K., Parker R., Berndt W.O., Philips T.D. Ochratoxin A induced teratogenesis in rats : partial protection by phenylalanine. Appl Environ Microbiol. 1984 ; $48: 1186-1188$.
7. Dwivedi P. and Burns R.B. Immunosuppressive Effects of Ochratoxin A in Young Turkeys. Avian Pathol. 1985 ; $14: 213-225$.

8. Bunge I., Dirheimer G. and Röschenthaler R. In vivo and in vitro Inhibition of Protein Synthesis in Bacillus stearthermophilus by Ochratoxin A. Biochem Biophys Res Commun. $1978 ; 83: 398-405$.

9. Dirheimer G. and Creppy E.E. Mechanism of Action of Ochratoxin A. In: Castegnaro, M., Plestina, R., Dirheimer, G., Chernozemsky, I.N., Bartsch, H. eds. Mycotoxins, Endemic Nephropathy and Urinary Tract Tumours ; vol. 115. IARC, Lyon. 1991 ; 171-186.

10. Pfohl-Leskowicz A., Grosse, Y., Kane A., Creppy E.E. and Dirheimer G. Differentiel DNA-adducts formation and disappearance in three mice tissues after treatement by the mycotoxin ochratoxin A. Mutat Res. 1993 ; 289 : 265-273.

11. Obrecht-Pflumio S., Chassat T., Dirheimer G., Marzin D. Genotoxicity of ochratoxin A by Salmonella mutagenicity test after bioactivation by mouse kidney microsomes. Gen Tox Env Mut. 1999 ; 446 : 95-102.

12. Krogh P. Role of ochratoxin in disease causation. Food Chem Toxicol. $1992 ; 30: 213-224$.

13. Castegnaro M., Bartsh H., Chernozemsky, I. Endemic Nephropathy and Urinary Tract Tumors in the Balkans. Cancer Res. 1987 ; 47 : 3608-3609. 
14. Bendele A.M., Carlton W.W., Krogh, P. and Lillehoj, E.B. Ochratoxin A. Carcinogenesis in the $(\mathrm{C} 57 \mathrm{BL} / 6 \mathrm{~J} \mathrm{x}$ C3H) F1 mouse. J Natl Cancer Inst, 1985 ; 75 : 733-742.

15. Mantle P.G., McHugh K.M., Adatia R., Gray T., Turner, D.R. Persistent karyomegaly caused by Penicillium nephrotoxins in the rat. Proc R Soc Lond Ser. 1991 ; $246: 251-259$.

16. Krogh P., Axelsen N.H., Elling F., Hansen N.G., Hald B., Jenson J.H., Larsen A.E., Madsen A., Mortensen H.P., Moler T., Peterson O.K., Burnkov V., Rostguard M., Aulund O. Experimental Porcine Nephropathy: Changes of renal function and structure induced by ochratoxin Acontaminated feed. Acta Pathol. Microbiol. Scand. Sect. A. $1974 ; 246: 1-21$.

17. Harwig J., Kuiper-Goodman T., Scott P.M. Micobial food toxicants: ochratoxins. In: Rechcigal, M.J. eds. CRC Handbook of Foodborne Diseases of Biological Origin CRC Press, FL. 1988 ; 193-238.

18. Tatu C.A., Orem W.H., Finkelman R.B., Feder G.L. The etiology of Balkan endemic nephropathy: still more questions than answers. Environ Health Perspect. 1998 ; $106: 689-700$.

19.Gaon J., Grigs R.C., Vasiljevic M.,Alibegovic S. Investigation of chronic nephropthy in Yougoslavia. I. Lead as a possible etiologic agent. Acta Med Iugosl. $1962 ; 16: 347-353$.

20.Piscator M., Kostial K., Plestina R. Urinary excretion of Cadmium and zinc among woman with endemic nephropthy. Trace Elem Med. $1984 ; 1: 134-138$.

21. Cosyns J.P., Jadoul M., Squifflet J. P; De Placon J. P., Ferluga D., Van Ypersele de Strihou C. Chinese herbs nephropathy: A clue to Balkan endemic nephropathy. Kidney Int. 1994 ; 45 : 1680-1688.

22. Vladisav S. Balkan endemic nephropathy. A Reapraisal after forty years. Med and Biol. 1999 ; 6 (1) : 53-58.

23. Maksimovic Z. J. Selenium deficiency and Balkan nephropathy. Kidney Int. $1991 ; 40$ (3) : S12-S14.

24. Krogh P., Hald B., Plestina R., Ceovic S. Balkan (endemic) nephropathy and foodborn ochratoxin A: preliminary results of survey of foodstuffs, Acta Pathol Microbiol Scand Sect A, 1977 ; 246 (Suppl.) : 1-21.

25. Stoev S.D., Daskalov H., Radic B., Domijan A.M., Peraica M. Spontaneous mycotoxic nephropathy in Bulgarien chikens with unclarified mycotoxin aetiology, 2000 ; Vet. Res. 33, 1, 83-93.

26. Petkova-Bocharova T. et Castegnaro M. Ochratoxin A in human blood in relation to Balkan endemic nephropathy and urinary tract tumours in Bulgaria, in: $M$. Castegnaro R. Plestina G. Dirheimer I.V. Chernozemsky and H. Bartsch. eds. Mycotoxin, endemic nephropathy and urinary tract tumours, IARC Scientific publications No. 115, International Agency for research on Cancer, Lyon, $1991 ; 135-137$.

27.Pfohl-Leskowicz A., Petkova-Bocharova T., Chernozemsky I.N., Castegnaro M. Balkan endemic nephropathy and associated urinary tract tumors : a review on aetiological causes and the potentiel role of mycotoxins. Food Addit Contam. 2002 ; 19 (3) : 282-302.

28.Pfohl-Leszkowicz A., Chakor K., Creppy E. E., Dirheimer G. DNA adduct formation in mice treated by ochratoxin A. Dans : Mycotoxins, Endemic Nephiupathy and Urinary Tract Tumours (M. Castegnaro, R. Plestina, G. Dirheimer, I. N. Chernozemsky, H. Bartsch eds). Lyon, 1991 ; IARC Sci. Publ. 115, 245-253.

29.Pfohl-Leszkowicz A., Grosse Y., Castegnaro M., Nicolov I. G., Chernozemsky I. N., Bartsch H., Betbeder A. M., Creppy E. E., Dirheimer G. Ochratoxin A - related DNA adducts in urinary tract tumours of Bulgarian suhjects. Dans Postlabelling Methods for Detection of DNA Adducts (D. H. Phillips, M. Castegnaro, H. Bartsch, eds). Lyon, 1993 ; IARC Sci. Publ. 115, 141-147.

30. Ceovic S., Hrabar A., Saric M. Epidemiology of Balkan Endemic Nephropathy. Fd Chem Toxic. 1992 ; 30 : 183-8.

31.Plestina R. Some features of Balkan endemic nephropathy. Fd. Chem. Toxic. $1992 ; 30: 177-181$.

32. Kuiper-Goodman T. and Scott P.M. Risk assessment of the mycotoxin ochratoxin A. Biomed. Environ. Sci. $1989 ; 2$ : 179-248.

33. Achour A., El May M., Bacha H., Hammami M., Maaroufi K., Creppy E.E. Néphropathies interstitielles chroniques. Approches cliniques et étiologiques: Ochratoxine A. In: Creppy, E.E., Castegnaro, M., Dirheimer, G. eds. Human Ochratoxicosis and its Pathologies, vol. 231. Colloque INSERM/John Libbey Eurotext, 1993 ; 227-234.

34. Vukelic M., Sostaric B., Belicza M. Pathomorphology of Balkan endemic nephropathy. Food Chem Toxicol. $1992 ; 30: 193-200$.

35. Maaroufi K., Achour A., Betbeder A. M., Hammami M., Ellouz F., Creppy E. E. Bacha H. Foodstuffs and human blood contamination by the mycotoxin ochratoxin A: correlation with chronic interstitial nephropathy in Tunisia, 1995; Arch. Toxicol. $69: 552-558$.

36. Maaroufi K., Achour A., Hammami M., EL May M., Betbeder A. M., Ellouz F., Creppy E. E. Bacha H. Ochratoxin $\mathrm{A}$ in human blood in relation to nephropathy in Tunisia. Human and Experimental Toxicol. 1995; 14 : 609-615.

37. Bauer J. et Gareis M. Ochratoxin A in the food chain, 1987 ; Z. Veterinärmed. B., 34, 613-627.

38. Gad S.C. et Neil C.S.(1982). Statistic for toxicologists. In principals and methods of toxicology, Wallace Hayes, A. ed. ; 273-279.

39. Scott P.M., Van Wabeek W., Kennedy B., Anyeti D. Mycotoxins ochratoxin $\mathrm{A}$, citinin and sterigmatocystin and toxigenic fungi in grains and other agricultural products. J Agr Food Chem. 1972 ; 20: 1103-1109.

40. Bacha H., Hadidane R., Regnault C., Ellouz F. and Dirheimer G. Mycotoxines et Mycotoxicoses en Tunisie. Cahiers Médicaux de Tunisie-Nutrition et santé. 1986 ; $49: 34-35$.

41. Hadidane R., Roger-Regnault C., Bouatour H., Ellouz F., Bacha H., Creppy E.E., Dirheimer G. Correlation between alimentary mycotoxin contamination and specific diseases. Hum Toxicol. $1985 ; 4$ : 491-501.

42. Krogh P. Environmental ochratoxin A and Balkan (endemic) nephropathy : evidence for support of causal relationship. In : Strahinjic S, Stefanovic V, ed. Endemic (Balkan) nephropathy . University Press, Nis, 1979 ; 35-3. 
43. Hult K., Plestina R., Habazin-Novak V., Radic B., Ceovic S. Ochratoxin A in human blood and Balkan endemic nephropathy. Arch Toxicol, 1982 ; $51: 313-21$.

44. Godin M., Fillastre JP., Simon P., Francois A., Le Roy F., Morin JP. Is ochratoxin A nephrotoxic in human beings ?Adv Nephrol $1996 ; 25: 187$.

45. Godin M., Fillastre J.P., Simon P., Francois A., Le Roy F., Morin J.P.. L'ochratoxine A est-elle néphrotoxique chez l'homme ? Flammarion Médecine -Sciences - Actualités néphrologiques, 1996 ; 225-50.

46. Aloui S. Rapport National du Ministère Tunisien de la Santé Publique : Epidemiologie de l'Insuffisance Rénale Chronique en Tunisie, 2000.
47. Radic B. Fuchs R., Peraica M., Lucic A. Ochratoxin A in human sera in the area with endemic nephropathy in Cratia. Tox Let. 1997, 91, 105-109.

48. Palli D., Miraglia M., Saieva C., Masala G., Cava E. et al. Serum levels of ochratoxin A in healthy Adults in Toscany. Can Epid. Bio and Prev. 1999, 8 (3).

49. Ozcelik N., Kosar A., Soysal D. Ochratoxin A in human serum samples collected in Ispartarke from health individuals and individuals suffering from different urinary disorders. Toxicol. Lett. 2001 ; 121, 1, 9-13.

50. MAAF. Asurvey of Human Exposure to Ochratoxin A. Food Surveillance information Sheet. $1999 ; 172$. 